\title{
Biotransformación de metales pesados presentes en lodos ribereños de los ríos Bogotá y Tunjuelo
}

\author{
Catherine Soto', Sonia Gutiérrez' ${ }^{1}$ Alexandra Rey-León', \\ Edwin González-Rojas ${ }^{1}$
}

\author{
1. Grupo de Investigación GRESIA, Universidad Antonio Nariño \\ Correspondencia: edwigonzalez@uan.edu.co
}

Recibido: 16-07-10 / Aceptado: 02-12-10

\begin{abstract}
Resumen
La presencia de metales pesados en los lodos ribereños de la zona media de los ríos Bogotá y Tunjuelo, procedentes de desechos de industriales, ha influenciado y permeado la calidad de los suelos en la Sabana de Bogotá. Esto se refleja en las pequeñas concentraciones presentes en vegetales que se utilizan para la alimentación humana, causando efectos nocivos para la salud. La biotransformación de estos metales es posible gracias a la presencia de algunas moléculas inorgánicas y de microorganismos capaces de interactuar, cambiando su biodisponibilidad en el medio. Esta investigación determinó la concentración de cromo, plomo y mercurio en la zona media de los ríos Bogotá y Tunjuelo, mediante la técnica de absorción atómica.

Los microorganismos se encuentran expuestos a la presencia de metales pesados, lo cual selecciona variantes capaces de tolerar sus efectos nocivos. El estudio de estas interacciones, con sus mecanismos de tolerancia, expone el potencial de éstos para ser utilizados en procesos biotecnológicos como la biorremediación de la contaminación ambiental por metales pesados. Mediante experimentos de laboratorio, se logró extraer cepas por cultivo directo y enriquecimiento selectivo y determinar su grado de compromiso en la biotransfomación y biodisponibilidad de metales como cromo, plomo y mercurio, concluyendo que uno de los géneros más comprometidos es el Micrococos, seguido por Pseudomonas.
\end{abstract}

Palabras clave: absorción atómica, biodisponibilidad, biotransformación, metales pesados, xenobióticos.

\begin{abstract}
Biotransformation of heavy metals present in mud bordering the Bogotá and Tunjuelo rivers

The presence of heavy metals in mud bordering the middle zone of Bogotá and Tunjuelo rivers, from industrial waste, has influenced and permeated the soil quality in the Bogotá plateau. This is reflected in small concentrations present in plants used for human consumption, causing adverse health effects. The biotransformation of these metals is possible due to the presence of some inorganic molecules and microorganisms capable of interacting, changing its bioavailability in the middle. In this investigation we determined the concentration of chromium, lead, and mercury by the atomic absorption technique, much used in the quantitative study of heavy metals.
\end{abstract}


Microorganisms are exposed to the presence of heavy metals, which select variants able to tolerate its adverse effects. The study of these interactions, their mechanisms of tolerance, exposes the potential for use in biotechnological processes such as bioremediation of environmental pollution by heavy metals. Through laboratory experiments, strains were able to be extracted by direct culture and selective enrichment and determine their degree of commitment to biotransformation and bioavailability of metals like chromium, lead, and mercury, concluding that one gender is more committed Micrococci, followed by Pseudomonas, without daring to mention more species due to the complexity and varieties identified in the environment.

Keywords: atomic absorption, bioavailability, biotransformation, heavy metals, xenobiotics.

\section{Introducción}

La contaminación ambiental generada por las actividades industriales, es uno de los problemas que cursa el planeta y que altera el equilibrio biológico de la Tierra. Los metales pesados son uno de los desechos contaminantes más tóxicos que se arrojan como desechos industriales; que aunque son imprescindibles para el desarrollo de los organismos, en cantidades excesivas pueden llegar a ser letales. Una vez emitidos se absorben muy fácilmente a través de las membranas biológicas por su elevada afinidad química a las proteínas no siendo de fácil degradación y dificultando su eliminación. Es así como se hace necesario contrarrestar estos contaminantes utilizando técnicas para su transformación y/o inactivación del ambiente.

La toxicidad de los metales pesados es muy alta. Su acción directa sobre los seres vivos ocurre a través del bloqueo de las actividades biológicas, es decir, la inactivación enzimática por la formación de enlaces entre el metal y los grupos sulfhidrilos $(-\mathrm{SH})$ de las proteínas, causando daños irreversibles en los diferentes organismos. Para que los metales pesados puedan ejercer su toxicidad, éstos deben encontrarse disponibles para ser captados por los seres vivos, es decir que el metal debe estar biodisponible. El concepto de biodisponibilidad se encuentra íntimamente relacionado con las condiciones físicoquímicas del ambiente, que determinan la especiación y por lo tanto la concentración de metal libre y lábil. Por ello es fundamental, al determinar el grado de contaminación por metales pesados de un ambiente, conocer su biodisponibilidad, es decir, la concentración de metal libre y lábil presente en la muestra $(1,2)$.

Una de las aplicaciones más recientes en la biotecnología es la corrección biológica que permite aborda en la mayor parte de los casos el tratamiento de suelos contaminados. Todas las interacciones entre los microorganismos y los metales u otros elementos como carbono, nitrógeno, azufre y fósforo son componentes fundamentales de los ciclos biogeoquímicos. Las interacciones metalmicrobiota son estudiadas en profundidad en el contexto de la biotecnología ambiental, con el objeto de implementar métodos de remoción, recuperación o detoxificación de metales pesados y radionúclidos (3-5).

Dependiendo del estado de oxidación que se presente un metal y la especie que esté conformando, un microorganismo puede realizar dos transformaciones posibles. Una correspondería a la movilización del metal, es decir el pasaje de un estado insoluble inicial (metales asociados a suelos, sulfuros u óxidos metálicos, por ejemplo) correspondiente a una fase sólida, a un estado soluble final, en fase acuosa. Este proceso se conoce con el nombre de lixiviación microbiana. El otro corresponde a la inmovilización del metal, es decir el pasaje de un estado soluble inicial en fase acuosa a uno insoluble final en fase sólida. A 


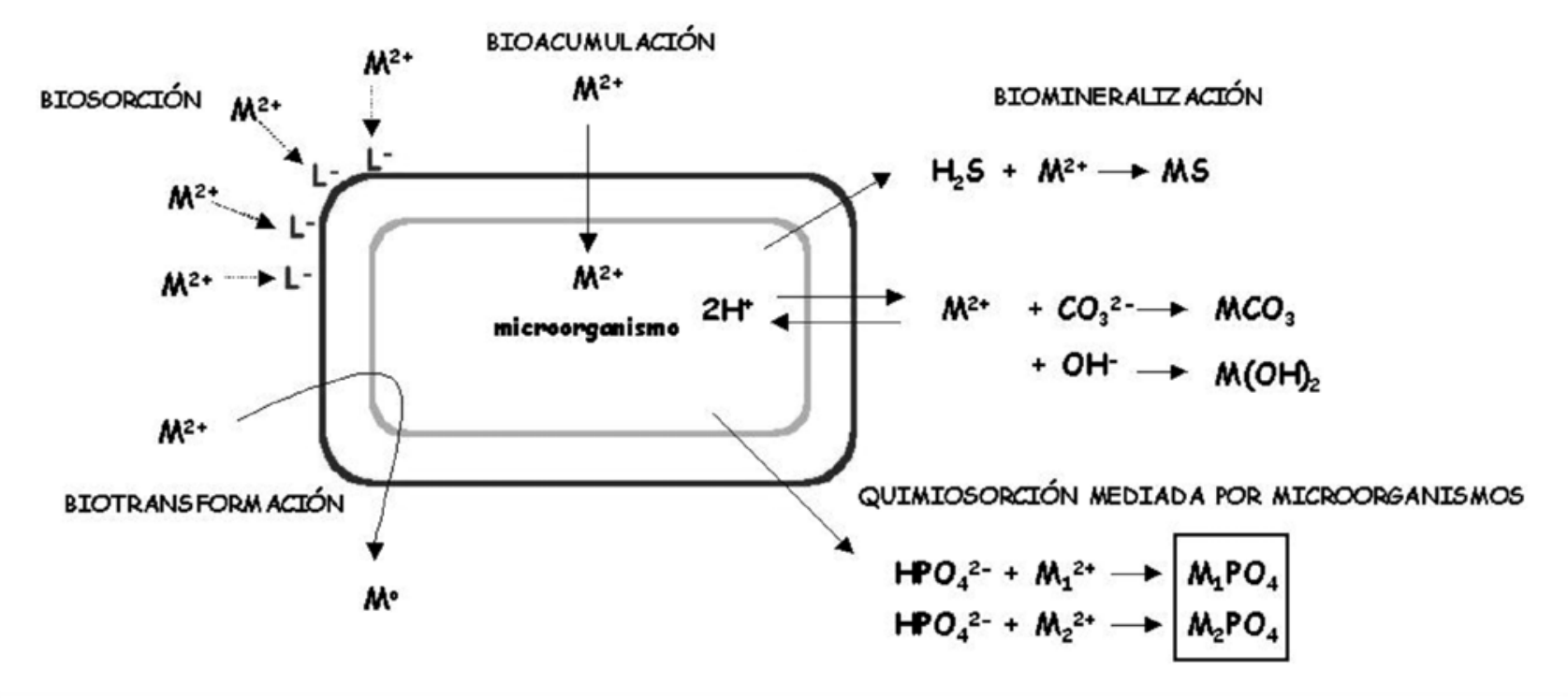

Figura 1: Interacción entre los metales pesados y los microorganismos. http://www.quimicaviva.qb.fcen.uba.ar/Actualizaciones/metales/metales.

su vez existen en la naturaleza diferentes mecanismos por los cuales la inmovilización del metal puede llegar a ocurrir (6).

El interés en descontaminar los suelos se produce debido a que el flujo del agua transporta la mayoría de los contaminantes y estos tienden a absorberse sobre las superficies presentes o forman fases líquidas, quedando atrapadas en la matriz sólida. Uno de los métodos de corrección biológica que se utiliza en la actualidad es la biorremediación, la cual surge como una rama de la biotecnología que busca resolver los problemas de contaminación (7).

Según la Environmental Protection Agency (EPA), la biorremediación se define como los procesos en los que se usan microorganismos o enzimas producidas por estos para transformar o degradar contaminantes tóxicos en los ecosistemas (8); esta estrategia biológica depende de las propiedades catabólicas que presentan los microorganismos, quienes utilizan los contaminantes para su desarrollo. Los contaminantes que se liberan en las superficies pueden ser transportados vertical y lateralmente a los suelos de aguas y eventualmente pueden ser inhalados o ingeridos através de aguas para el consumo humano, además pueden ser absorbidos por las plantas y los tejidos animales. Estos contaminantes son llamados también xenobióticos (término que se refiere a las sustancias artificiales que no pertenecen a los sistemas biológicos naturales) (9).

En la absorción microbiana, llamada bioacumulación, las especies metálicas son efectivamente retenidas al interactuar con los fosfatos, proteínas y lípidos en el citoplasma celular compitiendo con los iones sodio, potasio y calcio en los mecanismos biológicos; sus posibilidades de desarrollar una mayor capacidad absorbente dependen del tipo de microorganismo y su etapa de crecimiento (10). En la Figura 1 se esquematiza la interacción entre los metales pesados y los microorganismos, Figura 1.

Una característica química importante de las paredes celulares es que poseen igualmente grupos funcionales; las técnicas analíticas muestran que en ellas están presentes polímeros sustituidos con grupos aminos, amidos, carboxílicos, hidroxilicos, fosfatos. Una vez que la biomasa obtenida principalmente de los desechos de la industria que realizan procesos de fermentación ha sido secada, el residuo es un absorbente eficaz en la remoción de especies metálicas desde efluentes. Este proceso se denomina bioabsorción; igual que en la bioacumulación el tipo de microorganismo determina los biopolímeros presentes en la biomasa seca y asimismo la concentración de 
los grupos funcionales disponibles como sitios de adsorción. Este conocimiento ha permitido que otras investigaciones se orienten al uso directo de los biopolímeros, desarrollando aun más la biosorción como técnica de metales tóxicos desde efluentes mineros (10).

El ambiente más favorable para la mayor parte de los microorganismos (bacterias, hongos y algas) $(11,12)$ es:

- Temperatura: $15-45^{\circ} \mathrm{C}$.

- $\mathrm{pH}$ : entre 5,5 y 8,5 .

- Relación de nutrientes carbono: nitrógeno: fósforo de 120:10:1.

- Presencia de agentes oxidantes y/o reductores que permitan las reacciones metabólicas de los microorganismos.

Además de estas condiciones, para que la biorrecuperación sea efectiva, es necesario que los microorganismos sean los apropiados para el contaminante y que éstos no sean tóxicos para los microorganismos. El principal problema de los metales pesados en el ambiente es que no pueden ser biodegradados; sin embargo los microorganismos pueden interaccionar con ellos transformándolos los cuales obedecen a cambios en el estado de oxidación; esto influye de forma drástica en la movilidad del contaminante, ya que en algunos casos aumenta la solubilidad de los productos de alteración, favoreciendo así su eliminación del medio, y en otros casos disminuye produciéndose una inmovilización del contaminante. La elección entre una u otra transformación dependerá de sí se pretende eliminar la contaminación o sí se quiere evitar su llegada a zonas específicas. Además los microorganismos pueden actuar y biodegradar la materia orgánica y los compuestos orgánicos (ácidos húmicos principalmente), modificando igualmente su movilidad.

Las reacciones responsables de los procesos de biotransformación y biodegradación están relacionadas con el metabolismo de los microorganismos existentes. Para que dichos microorganismos puedan crecer y desarrollar sus funciones vitales necesitan un aporte de nutrientes, básicamente carbono, nitrógeno y fósforo; así como donantes y aceptores de electrones (13). Estos últimos son imprescindibles como fuente de energía, ya que la transferencia catalizada de electrones entre donantes y receptores libera la energía requerida para que se produzcan las reacciones bioquímicas vitales. Algunos metales pesados pueden actuar como donantes y aceptores de electrones, o como fuente de energía, y por ello es posible su biotransformación. Los organismos que utilizan metales como fuentes principales de energía son los denominados chemolitoautótrofos.

Las principales transformaciones de los metales pueden ser directas, por medio de cambios en el estado de valencia cuando actúan como donantes o receptores de electrones e indirectas, por medio de agentes oxidantes y reductores producidos por los microorganismos y que son responsables de cambios en el pH y del potencial redox (11). En la respiración aerobia los organismos chemolitoautótrofos utilizan $\mathrm{CO}_{2}$ como fuente de alimentación y de energía. En la respiración anaerobia, los procesos están condicionados por el tipo de aceptor de electrones, ya que no existe oxígeno disponible para hacer dicha función. Los principales aceptores de electrones en medios anaerobios son $\mathrm{CO}_{2} ; \mathrm{NO}_{3}^{-} ; \mathrm{SO}_{4}^{2-}$ y Fe ${ }^{3+}$.

Las formas oxidadas de los metales son altamente solubles y por lo tanto móviles, en las aguas subterráneas en condiciones aerobias. Su reducción enzimática produce formas reducidas insolubles, lo que provoca su precipitación intra o extracelular (11). Además de la reducción enzimática directa de metales pesados, algunos microorganismos, como las bacterias reductoras de metales y las bacterias reductoras de sulfatos, pueden llevar a cabo la reducción indirecta de especies oxidadas solubles. Esto se produce acoplando la oxidación de compuestos orgánicos a la reducción del $\mathrm{Fe}^{3+}, \mathrm{Mn}^{4+}$ y $\mathrm{SO}_{4}^{2-}$. Las especies reducidas $\mathrm{Fe}^{2+}$, $\mathrm{Mn}^{2+}$ y $\mathrm{H}_{2} \mathrm{~S}$, interaccionan químicamente con los metales contaminantes solubles produciendo especies multicomponentes insolubles que precipitan.

La solubilización de metales bioabsorbidos y coprecipitados puede producirse por procesos microbianos tanto de forma directa como indirecta, aunque la solubilización de metales pesados a 
partir de coprecipitados con las fases minerales del acuífero, requiere una solubilización parcial previa de dichas fases minerales, óxidos principalmente. La reducción directa de óxidos de hierro por bacterias reductoras de metales, favorece la solubilización de un gran rango de metales pesados que pudieran estar coprecipitados en dichos óxidos. Asimismo, la creación de ácidos orgánicos durante el metabolismo de los microorganismos, es capaz de bajar el $\mathrm{pH}$ del sistema, hasta valores que permitan interferir con las cargas electrostáticas que mantienen unidos los metales pesados a la superficie de los óxidos e hidróxidos de hierro y ser desplazados por los iones de hidrógeno liberados al sistema.

\section{Materiales y métodos}

Basándonos en los reportes de algunos trabajos de grado de Universidades como la Distrital y los Andes, entre otras, y los informes de la Contraloría Distrital y la Secretaria Distrital de Ambiente, se determinó la zona media del rio Tunjuelo y la zona media del rio Bogotá. El proyecto identificó dos ambientes típicamente contaminados (suelos y lodos) con metales pesados $(\mathrm{Cr}, \mathrm{Pl}, \mathrm{Hg})$, en los cuales se tomaron muestras en forma continua durante los 12 meses en que se desarrolló el proyecto, para lo cual se realizaron visitas y muestreos directos.

Partiendo de la determinación de la zona de muestreo y haciendo una caracterización física, química y climática de la zona, se pudo estandarizar los protocolos microbiológicos a seguir en el laboratorio, y se utilizó la absorción atómica como técnica para la determinación de estos metales pesados. Con la zona de muestreo estudiada, los protocolos microbiológicos establecidos, se procedió a la toma de las muestras de los lodos semilíquidos dadas las condiciones de las riveras de estos dos ríos. Se tomaron muestras por duplicado en frascos de $500 \mathrm{~mL}$, color ámbar y conservados a $4^{\circ} \mathrm{C}$, (una para química y otro para microbiología). Las muestras fueron recogidas con herramientas manejables (paletas o espátulas) previamente esterilizadas en el laboratorio o bien a la llama inmediatamente antes de la toma. En el caso de los suelos siempre se trató de muestras superficiales $(20$ o $30 \mathrm{~cm})$.

El proceso de preparación pasó por un pequeño cribado manual para retirar trozos de roca y vegetales de modo que la muestra presentara un aspecto homogéneo, sin fragmentos superiores a $5 \mathrm{~mm}$. Es importante conseguir una solución convenientemente clara, representativa y reproducible para los cultivos en placa. Para ello, a un peso de cada muestra (10 g) se le añadió una cantidad de un agente disgregante (pirofosfato de sodio $-\mathrm{Na}_{4} \mathrm{P}_{2} \mathrm{O}_{7} \cdot 10 \mathrm{H} 2 \mathrm{O}$ al $0,1 \%$ ) en una relación constante volumen/peso de 2,5 (14). El recipiente estéril se agitó, machacando las partículas de suelo con una varilla de vidrio también estéril hasta reposar y se observó el grado de dispersión alcanzado. El proceso se detuvo cuando se dispersaron por completo las partículas mayores. Este procedimiento actúa a escala macroscópica (15) la dispersión de las partículas de suelo y la ruptura de agregados que pudieran disminuir la eficiencia de la extracción de los microorganismos. En el ámbito microscópico se consigue una desorción de las células suficiente para conseguir una suspensión microbiana.

Las muestras se sometieron a análisis química para poder establecer el nivel de contaminación de estos lodos por $\mathrm{Cr}$ hexavalente, $\mathrm{Pl}$ y $\mathrm{Hg}$. Las muestras microbiológicas se concentraron mediante centrifugación a $12000 \mathrm{rpm}$ durante 5 minutos en forma fraccionada y se tomó el sobrenadante, llevando a un volumen final de $50 \mathrm{~mL}$, para iniciar el proceso de diluciones y siembra en medio TSA e incubarlos por 48 horas a una temperatura de $25^{\circ} \mathrm{C}$. Se realizaron repiques y aislamiento de los microorganismos presentes (biotransformantes y biotolerantes), para ser sometidos a pruebas bioquímicas (16) iníciales de identificación y establecer las técnicas de enriquecimiento selectivo en matraces de $250 \mathrm{~mm}$ con medio de cultivo (lodo estéril) a $5000 \mathrm{rpm}$ y $25^{\circ} \mathrm{C}$ durante siete días, al cabo de los cuales se tomó $1 \mathrm{~mL}$ para diluciones (17), siembra, recuento aislamiento e identificación $(15,18,19)$.

Todos los procedimientos que se pueden usar para el cálculo del número de microorganismos viables en una muestra natural tienen implicitos errores; los recuentos en placa no son excepción. 
Efectivamente, el número de microorganismos que se pueden aislar con esta técnica es muy inferior al total de los presentes en la misma muestra; tanto es así que, según los casos, se habla de un $10 \%$ a $15 \%$ del total $(20,21)$. Se tomaron $25 \mathrm{~mL}$ del matraz y se resembró en un nuevo matraz medio de cultivo líquido (lodos esterilizados), repitiendo tres veces el procedimiento anterior, (enriquecimiento selectivo).

Se implementaron nuevos matraces de $250 \mathrm{~mL}$, con medio de cultivo estéril, (lodos analizados químicamente para garantizar la ausencia de metales pesados) suplementados con adición de concentraciones especificas de $\mathrm{Cr}, \mathrm{Pl}$ y $\mathrm{Hg}$ para observar el comportamiento de los microorganismos aislados (biotolerantes y biotransformadores).

Es importante señalar que el contenido de cada matraz fue enviado a análisis químico para hacer seguimiento a la biotransformación de cada uno de estos metales. Se utilizaron técnicas de biología molecular, para la identificación de los microorganismos así como el análisis de sus relaciones filogenéticas, mediante la secuenciación del ARN ribosómico (rRNA), o de los genes que se codifican, rDNA. El proceso completo pasó por fases de amplificación (PCR) del gen del RNAr $16 S$, secuenciación y finalmente comparación con las secuencias de referencia en las bases de datos existentes (15).

La aportación principal de estas técnicas en el campo de biorremediación es la identificación precisa de los microorganismos implicados en la biotransformación. Los árboles filogenéticos que se pueden elaborar con esta metodología permiten conocer las relaciones de las bacterias detectadas con otros grupos microbianos, incluso sin haber sido cultivadas previamente.

Los métodos utilizados para identificar las cepas aísladas tras diversas fases de cultivo fueron: obtención de $\mathrm{ADN}$ (22), amplificación-purificación: mediante el uso del kit GFX PCR DNA and Gel Band purification (Amersham Bioscience) según el protocolo del fabricante y leyendo el ADN obtenido en $60 \mu \mathrm{L}$ de agua destilada; secuenciación: $10 \mu \mathrm{L}$ de
ADN purificado (a una concentración aproximada de $30 \mathrm{ng} / \mu \mathrm{L}$ ) mediante el uso del kit Thermo sequenase dye terminator cycle sequencing premix (Amersham Bioscience) y $5 \mu \mathrm{L}$ del cebador $\mathrm{pH}$ a una concentración de $5 \mathrm{pM}$ en un secuenciador automático de ADN Multigene Termal Cycler TC9600; análisis de las secuencias de ADN: las secuencias obtenidas se introdujeron en el programa BLAST (NCBI) que busca homologías al nivel de nucleótidos o de aminoácidos con secuencias presentes en bases de datos (23).

\section{Resultados}

Los resultados de los análisis químicos de los lodos de ambos ríos (Bogotá y Tunjuelo), que fueron llevados a cabo en el Laboratorio de Química de la Universidad de Oviedo, España, fueron los siguientes:

- Cromo hexavalente mayor a $0.005 \mathrm{mg}$ de $\mathrm{Cr} / \mathrm{L}$.

- Plomo: mayor a $0.049 \mathrm{mg}$ de Pl/L

- Mercurio: mayor a $0.001 \mathrm{mg} \mathrm{de} \mathrm{Hg} / \mathrm{L}$.

La evidencia de su presencia es superior a lo establecido por la norma. Igualmente a las muestras se les realizó pruebas para determinar su $\mathrm{pH}$ (Laboratorio de Microbiología de la Facultad de Ingeniería Ambiental de la Universidad Antonio Nariño), obteniéndose los siguientes resultados:

- Muestra A1 (azul): 7.17

- Muestra A1 (blanca): 7.04

- Muestra B1: 6.83.

Los resultados son la expresión de un ANAVA aplicado al número total de muestras y su ponderación. Igualmente, de estas muestras, tomadas en forma consecutiva (cada 15 días), se obtuvieron diferentes colonias, el predominio fenotípico y comportamiento a la prueba bioquímica de Gram y oxidasa se muestran en la Tabla 1.

Se realizaron repiques para hacer pruebas de identificación mediante la técnica de CRYSTAL RAPID, determinándose de esta forma la presunta presencia de los siguientes microorganismos: Corynebacterium genitalium, Corynebacterium 
Tabla 1. Características morfológicas de los microorganismos aislados.

\begin{tabular}{|c|c|c|c|c|c|c|c|c|}
\hline Colonias & Al & A2 & A3 & A4 & A5 & A6 & A7 & A8 \\
\hline $\begin{array}{c}\text { Color } \\
\text { aparente }\end{array}$ & Beige & Blancas & Blancas & Amarilla & Rosada & Amarilla & Rosadas & Blancas \\
\hline Morfología & Cocobacilos & $\begin{array}{c}\text { Bacilos } \\
\text { esporulados }\end{array}$ & Bacilos & Bacilos & Bacilos & Cocobacilo & Cocobacilos & Cocos \\
\hline Gram & Negativo & Positivos & Negativo & Negativo & Negativo & Negativo & Negativo & Positivos \\
\hline Oxidasa & Negativo & Positivas & Negativa & Negativo & Negativo & Positiva & Negativo & Positivo \\
\hline
\end{tabular}

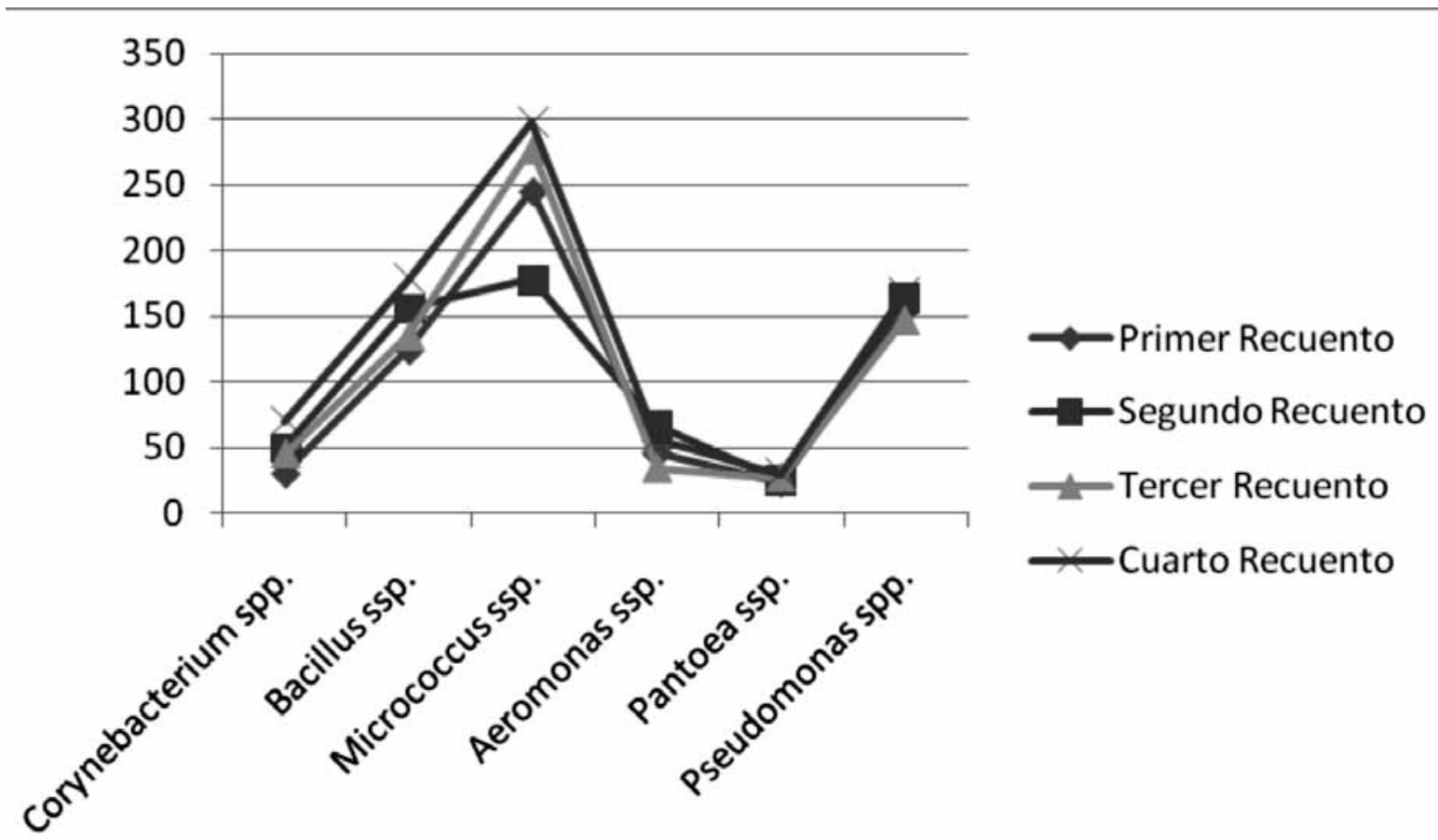

Figura 2. Resultado de dominancia de diferentes generos de microorganismos en Recuentos Directos en Medio TSA.

diphteriae, Bacillus megaterium, Micrococcus sedentarius, Bacillus meguterium, Aeromonas hydrophila, Aeromonas spp., Pantoea agglomerans y Pseudomonas spp. Los anteriores resultados son producto de la ponderación del recuento directo de la siembra en medio de cultivo TSA, por triplicado:

La literatura reporta la relación de algunos de estos microorganismos en la presencia de metales pesados, bien sea como tolerantes o como biotransformadores, y entre ellos tenemos como ejemplo a Corynebacterium genitalium, que presenta sensibilidad al cromato y resistencia al mercurio, resistencia al plomo,
Gram positivo; Corynebacterium diphteriae, ha sido aislado de suelos contaminados con metales pesados (cromo VI); Aeromonas hydrophila: Gram negativa, sensibilidad al cromato, resistencia al mercurio y resistencia al plomo; Pantoea agglomerans, crece entre $18^{\circ} \mathrm{C}-25^{\circ} \mathrm{C}$, bacilos Gram negativo, aislado en suelos contaminados con cromo VI; Pseudomonas sp, resistencia a cromo VI $(11,24)$, Figura 2.

Igualmente se realizaron diferentes aislamientos por enriquecimiento, y las especies que demostraron que intervienen, de alguna forma, en tolerancia en el proceso de biotransformación se relacionan en las Tablas 2-6. 
Tabla 2. Primer enriquecimiento.

\begin{tabular}{|c|c|c|c|}
\hline MUESTRA AI-I & MUESTRA AI-2 & MUESTRA AI-3 & MUESTRA AI-4 \\
\hline $\begin{array}{l}\text { Colonias blancas: Bacilos } \\
\text { Gram positivos } \\
\text { Corynebacterium genitalium/ } \\
\text { diphteriae }\end{array}$ & $\begin{array}{l}\text { Colonias blancas: Cocos Gram } \\
\text { positivos } \\
\text { Micrococcus sedentarius }\end{array}$ & $\begin{array}{l}\text { Colonias blancas: } \\
\text { Cocos Gram positivos } \\
\text { Micrococcus sedentarius }\end{array}$ & $\begin{array}{l}\text { Colonias amarillas: Bacilos } \\
\text { Gram positivos } \\
\text { Corynebactyerium }\end{array}$ \\
\hline $\begin{array}{l}\text { Colonias amarillas: Bacilos } \\
\text { Gram positivos } \\
\text { Bacillus, spp }\end{array}$ & $\begin{array}{l}\text { Colonias beige: Cocos Gram } \\
\text { positivos } \\
\text { Corynebacterium genitalium }\end{array}$ & $\begin{array}{l}\text { Colonias amarillas: Bacilos } \\
\text { Gram positivos }\end{array}$ & $\begin{array}{l}\text { Colonias blancas: Bacil os Gram } \\
\text { positivos }\end{array}$ \\
\hline & $\begin{array}{l}\text { Colonias crema: Cocos Gram } \\
\text { positivos } \\
\text { Micrococcus sedentarius } \\
\text { Colonias amarillas: Bacilos } \\
\text { Gram positivos } \\
\text { Pantoae spp. }\end{array}$ & & $\begin{array}{l}\text { Colonias beige: Cocos Gram } \\
\text { positivos }\end{array}$ \\
\hline MUESTRA BI-I & MUESTRA BI-2 & MUESTRA BI-3 & MUESTRA BI-4 \\
\hline $\begin{array}{l}\text { Colonias blancas: Bacilos } \\
\text { Gram positivos } \\
\text { Corynebacterium genitalium/ } \\
\text { diphtheriae }\end{array}$ & $\begin{array}{l}\text { Colonias blancas: Bacilos } \\
\text { Gram positivos }\end{array}$ & $\begin{array}{l}\text { Colonias beige: } \\
\text { Bacilos Gram positivos }\end{array}$ & $\begin{array}{l}\text { Colonias blancas: Cocos Gram } \\
\text { positivos } \\
\text { Micrococcus sedentarius }\end{array}$ \\
\hline $\begin{array}{l}\text { Colonias beige: Bacilos Gram } \\
\text { positivos } \\
\text { Corynebacterium genitalium / } \\
\text { especies de Corynebacterium }\end{array}$ & $\begin{array}{l}\text { Colonias beige: Bacilos Gram } \\
\text { positivos } \\
\text { Corynebacterium pseudogenitalium }\end{array}$ & & $\begin{array}{l}\text { *Colonias beige: } \\
\text { Bacilos medianos Gram positivos }\end{array}$ \\
\hline $\begin{array}{l}\text { Colonias transparentes: } \\
\text { Bacilos Gram positivos. }\end{array}$ & $\begin{array}{l}\text { Colonias crema: Cocos Gram } \\
\text { positivos } \\
\text { Micrococcus sedentarius }\end{array}$ & & \\
\hline
\end{tabular}

\section{Discusión}

En el momento de realizar el análisis de los resultados se debe tener una consideración importante, dado que, muy a pesar de los grandes adelantos que se presentan en biología molecular, y el grado de las secuencias con las diferentes bases de datos existentes, hay que considerar que tan solo se ha llegado a aislar el $10 \%$ de los microorganismos existentes en el 
Tabla 4. Del tercer enriquecimiento tenemos.

\section{MUESTRA Cl MUESTRA Cl}

Agar Nutritivo

\section{Colonia blancas}

Corynebacterium
Agar Sabouraud

Colonia amarillas

Pantoea aglomerans
Agar Nutritivo

Colonia blancas

Aeromonas hydrophila
Agar Sabouraud

\section{Colonia amarillas}

Corynebacterium genitalium/

diphtheriae

Tablas 5 y 6. Resultados del cuarto enriquecimiento.

\begin{tabular}{llll|}
\multicolumn{1}{c|}{ MUESTRA AI-I } & \multicolumn{1}{c|}{$\begin{array}{c}\text { MUESTRA } \\
\text { Al-2/A-2 }\end{array}$} & MUESTRA AI-3/A3 & \multicolumn{1}{c|}{$\begin{array}{c}\text { MUESTRA } \\
\text { Al-4/A4 }\end{array}$} \\
\hline $\begin{array}{l}\text { Colonia blanca } \\
\text { Corynebacterium genitalium/ } \\
\text { diphtheriae }\end{array}$ & $\begin{array}{l}\text { Colonia blanca } \\
\text { Micrococcus sedentarius }\end{array}$ & $\begin{array}{l}\text { Colonia blanca } \\
\text { Micrococcus sedentarius }\end{array}$ & $\begin{array}{l}\text { Colonia amarilla } \\
\text { Pantoe }\end{array}$ \\
$\begin{array}{l}\text { Colonia amarilla } \\
\text { Pseudomonas }\end{array}$ & $\begin{array}{l}\text { Colonia beige } \\
\text { Corynebacterium genitalium }\end{array}$ & & \\
& & & \\
& &
\end{tabular}

Colonia crema

Micrococcus sedentarius

Colonias amarillas: Bacilos Gram

positivos

Pseudomonas

\begin{tabular}{|c|c|c|c|}
\hline MUESTRA BI-I & MUESTRA BI-2 & MUESTRA BI-3 & MUESTRA BI-4 \\
\hline Colonia blanca & Colonia beige & Colonia beige & Colonia blanca \\
\hline Corynebacterium genitalium/diphtheriae & Corynebacterium pseudogenitalium & & Micrococcus sedentarius \\
\hline $\begin{array}{l}\text { Colonia beige } \\
\text { Corynebacterium genitalium/ especies de } \\
\text { Corynebacterium }\end{array}$ & $\begin{array}{l}\text { Colonia crema } \\
\text { Micrococcus sedentarius }\end{array}$ & & \\
\hline
\end{tabular}

medio ambiente y por esto mismo las bases de datos reportan géneros y especies en esta misma proporción. Debido a las anteriores consideraciones se reportan los resultados de semejanza expresados en género más no en especie.

De los resultados, tanto de los aislamientos por triplicado directos, como de los diferentes enriquecimientos, evaluadios mediante un análisis de varianza, se obtuvo la siguiente información: de los enriquecimientos directos se presenta una estandarización cuantitativa en la presencia de los géneros Corynebacterium, Bacillus, Aeromonas, Pseudomonas, Micobacterium, Aspergillus, sin embargo la presencia del género Micrococcus, pasó casi que inadvertida, lo cual fue cambiando a través de los 


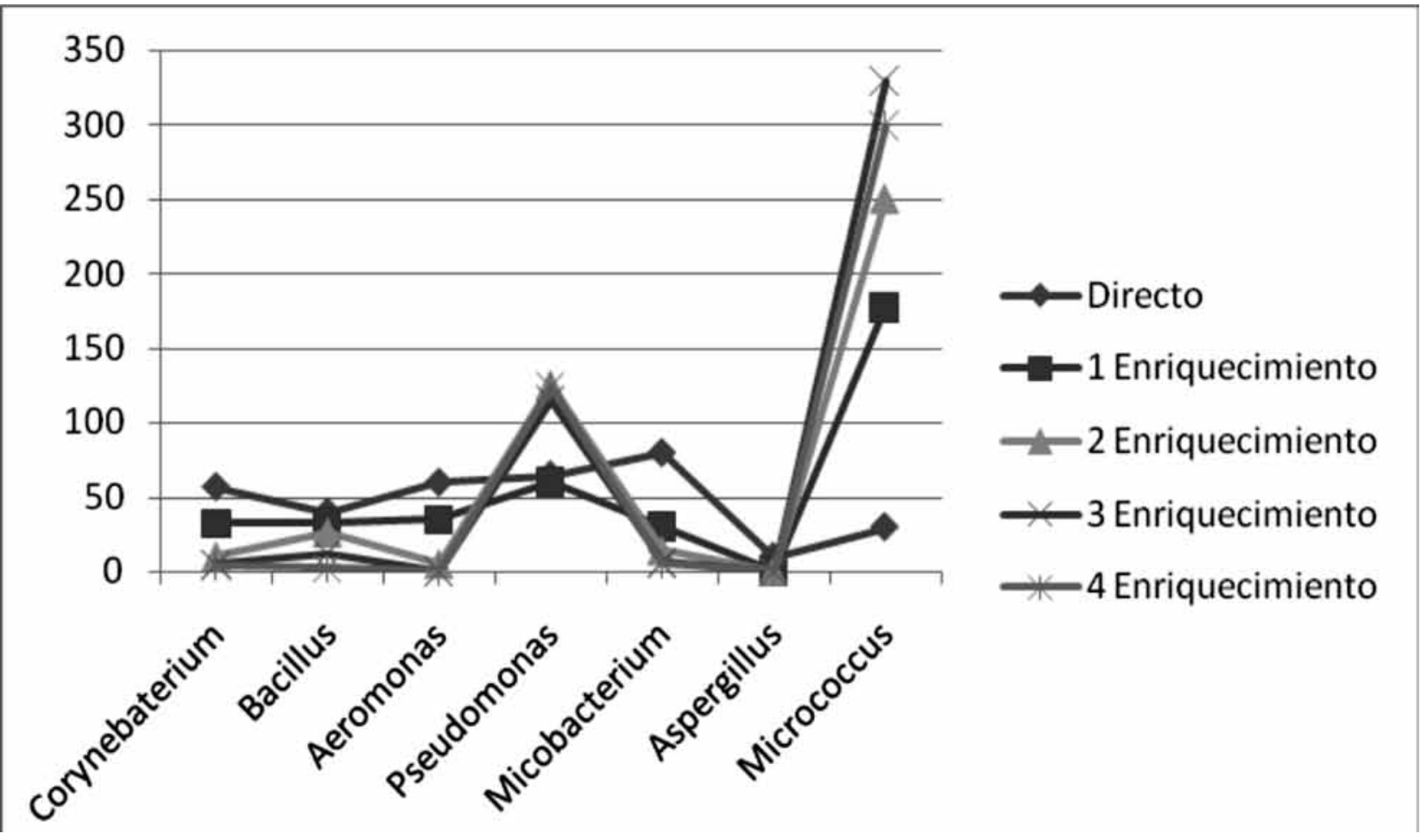

Figura 3. Géneros predominantes en la biotransformación de los metales $\mathrm{Cr}$, Pl y Hg.

enriquecimientos selectivos y de los enriquecidos con metales, llegando a dominar con su presencia.

Con lo anterior, se ratifica la importancia de no descartar ningún microorganismo de los aislamientos directos, los cuales pueden aparecer en forma poco significativa frente a las dominantes que pueden estar degradando metabolitos intermedios $\mathrm{u}$ otros sustratos presentes producto de la degradación del mismo medio, Figura 3.

La biotransformación de estos metales, tanto en los enriquecimientos con medio de cultivo con lodos como en los que se les adicionó $\mathrm{Cr}$, Ply $\mathrm{Hg}$ a los lodos esterilizados, fue casi total, demostrando la eficiacia de todos los generos presentes bien sea trasformado los metales directamente o suplementando otros bioelementos para facilitar este proceso.

\section{Agradecimientos}

A la Dirección Nacional de Investigaciones de la Universidad Antonio Nariño, por la financiación de este proyecto. Igualmente al laboratorio de Química de la Universidad de Oviedo por los análisis químicos de las muestras.

\section{Referencias}

1. Brown GE Jr, Foster AL, Ostergren JD. Mineral surfaces and Bioavailability of Heavy Metals: a Molecular-scale Perspective. Proc. Natl. Acad. Sci. USA.1999;96:3388-3395.

2. Mason R, Lawrence A. Concentration, Distribution, and Bioavailability of Mercury and Methylmercury in Sediments of Baltimore Harbor and Chesapeake Bay, Maryland, USA. Environ Toxicol Chem. 1999;18:2438-2447.

3. Lovley DR. Environmental Microbe-Metal Interactions. Ed. American Society for Microbiology. Washington D.C. 2000.

4. Gadd GM. Bioremedial potential of microbial mechanisms of metal mobilization and immobilization. Curr Opin Biotechnol. 2000;11:271-279.

5. Valls M, de Lorenzo V. Exploiting the genetic and the biochemical capacities of bacteria for the remediation of heavy metal pollution. FEMS Microb Rev. 2002;26:327-338.

6. Vullo, Diana. Microorganismos y metales pesados: una interacción en beneficio del medio ambiente. Química Viva. 2003;2:93-104.

7. Rittmann $\mathrm{B}, \mathrm{McC}$ arty P. Biotecnologìa del medio ambiente principios y aplicaciones. Mexico; Ed. Mc Graw Hill. 2001.

8. Sylvia D, Hartel P, Fuhrmann J, Zuberer D. Principles and applications of soil Microbiology. $2^{\circ}$. ed. Pearson Prentice Hall. 2005.

9. Coyne M. Soil Microbiology: an exploratory approach. Delmar Publisher Unite States of America. 1999.

10. Flores J, Arrascue M; Tapia N, Maldonado H. Biorremediación de métales tóxicos en efluentes mineros aplicando biosorción. Rev Inst investig Fac minas metal cienc geogr. 2001;4:46-51.

11. López J, García O, Grima J, Ballesteros B, Pérez O. Técnicas de Biorrecuperación in situ en acuíferos contaminados por metales pesados. Instituto geológico y minero de España. 233 -243. 
12. McCullough J, Hazen T, Benson S, Metting F, Palmisano A. Bioremediation of metals and radionucleids. 1999. En: http://www. escholarship.org/uc/item/8s57v0cs?display=all

13. Van Cauwenberghe y Roote, Technology Overview reports in situ. Bioremediation. 1998. En: http://www.gwrtac.org.

14. Fredrickson JK. \& D.L. Balkwill. Sampling and enumeration techniques. In: Burlage R S, Atlas R, Stahl D, Geesey G \& Sayler G (Eds) Techniques in Microbial Ecology (pp 239-254), Oxford University Press, Nueva York. 1998.

15. Ogram, A. \& X. Feng. Methods of soil microbial community analysis. In: Hurst CJ (Ed) Manual of Environmental Microbiology. ASM Press, Washington, D.C. (1997).

16. Díaz R, Gamazo C, López-Goñi I. Manual práctico de microbiología. Masson S.A., Barcelona.1999.

17. Madigan MT, Martinko J, . Parker. Brock, Biología de los Microorganismos. Prentice Hall, Madrid (2000).

18. Atlas, R.M. Handbook of media for environmental microbiology. CRC Press, Boca Raton (1995).
19. Riser-Roberts E Remediation of petroleum contaminated soils. CRC Press. Lewis publishers, NY (1998).

20. Amann RI, Ludwig W, Schleifer KH. Phylogenetic identification and in situ detection of individual microbial cells without cultivation. Microbiol Rev. 1995;59:143-169.

21. Wrenn BA, Venosa AD. Selective enumeration of aromatic and aliphatic hydrocarbon degrading bacteria by a most-probable-number procedure. Can J Microbiol. 1996;42:252-258.

22. Peláez Andrés, A.I. El gen recR de Streptomyces, caracterización estructural y funcional. Tesis doctoral, Universidad de Oviedo (2000).

23. Altschul SF, Madden TL, Schäffer AA, Zhang J, Zhang Z, Miller W, Lipman DJ. Gapped BLAST and PSI-BLAST: a new generation of protein database search programs. Nucleic Acids Research. 1997;25:3389-3402.

24. Atlas, R.M. \& R. Bartha. Ecología Microbiana \& Microbiología Ambiental. Pearson Educación, Madrid (2001). 